\title{
Hollow-Core Optical Fibers
}

\author{
Walter Belardi \\ CNRS, UMR 8523-PhLAM-Physique des Lasers Atomes et Molécules, Université de Lille, F-59000 Lille, France; \\ walter.belardi@univ-lille.fr \\ Received: 15 May 2019; Accepted: 22 May 2019; Published: 24 May 2019 \\ check for \\ updates
}

The possibility of guiding light in air has fascinated optical scientists and engineers since the dawn of optical fiber technology [1]. However, a remarkable progress in this area has been achieved "only" twenty years ago, when the first fabrication of a hollow-core photonic crystal fiber capable of delivering light over a length of few centimeters [2] gave rise to an increased interest in the field. Then, first the $20 \mathrm{~dB} / \mathrm{km}$ attenuation barrier was overcome [3] and, few years later, the lowest loss $(1.2 \mathrm{~dB} / \mathrm{km})$ hollow-core optical fiber $(\mathrm{HC})$ was realized [4].

Since the beginning of this century, HCs have attracted the attention of a large worldwide research community working on the design, fabrication and device implementation, entering almost any specific application field of optics (from medicine [5] to security [6], telecommunication [7], industrial processing [8], instrumentation [9], biology [10], and so on and so forth). In parallel with the increased number of applications, still major advances are being made on the optimization of the hollow-core fiber designs and on the study of its underlying guiding properties, as well as in the use of different materials or fabrication techniques, which, in turn, are providing even more ways of exploitation of this technology and new technical challenges.

This special issue of Fibers wanted to ride the wave of this renewed interest in the field of hollow-core optical fibers by providing an overview of the recent progress in this field as well as an updated and indicative sample of current research activities worldwide.

Thus, the issue includes three outstanding reviews by leading institutions in the field of hollow-core optical fibers. The review Hollow-Core Fiber Technology: The Rising of "Gas Photonics" [11] by the University of Limoges (France) and the University of Modena and Reggio Emilia (Italy) moves from their first discovery and development of the Inhibited-Coupling hollow-core optical fiber to its application to gas photonics. It is an extremely rich, deep and detailed trip offered by some of the most renowned scientists in the field that highlights their key achievements in both design and fabrication developments, and, in particular, shows how this gave rise to the exploitation of gas/light interaction in an unprecedented way.

The review Revolver Hollow-Core Optical Fibers [12] by the Fiber Optics Research Center (FORC), in Moscow, focuses on their specific simplified designs (HCs with only a single ring of tubular tubes in the cladding area), first pioneered and developed in their institution. Most properties, applications and fabrication approaches of this specific fiber type are addressed and discussed in all spectral domains. The review is not limited to silica glass, but also covers their demonstration of chalcogenide hollow-core optical fibers for the longer wavelength ranges.

The material and fabrication aspect is the object of the third review, 3D-Printed Hollow-Core Terahertz Fibers [13] by Instituto Tecnologico de Aerenoautica, Instituto de estudos avançados and Universidade Estadual de Campinas (UNICAMP), in Brazil. The realization and characterization of polymer-based HCs, in combination with 3D-printing fabrication, approaches is widely discussed. The review shows how the field of HCs is expanding also to the terahertz spectral regime and how it is starting to profit of the opportunities offered by the 3D-printing techniques.

After this overview on the last generation of hollow-core optical fibers, this special issue includes seven original contributions by scientists addressing current relevant issues involved in the design and application aspects of HCs. 
On the design aspect, the paper Effect of Nested Elements on Avoided Crossing Between the Higher-Order Core Modes and the Air-Capillary Modes in Hollow-Core Antiresonant Optical Fibers [14], by the Research Technology Organization of Photonics Bretagne (PERFOS), in Lannion (France), deals with the extremely important problem of mono-modality in the most advanced forms of HCs. The accurate numerical analysis made by the author provides an important insight in order to understand which HC geometry to use and how to simplify the analysis of its properties.

In the same way, the original paper Understanding Dispersion of Revolver-Type Anti-Resonant Hollow-Core Fiber [15] by the Leibnitz Institute of Photonic technology and the University of Jena, in Jena (Germany), is about the full comprehension of the dispersion properties of anti-resonant HCs, which is essential for applications involving high optical power and short pulse duration. Aside from providing useful analytical approximations, the authors perform a series of numerical simulations showing how the group velocity dispersion changes with the HC geometry.

Structure optimization is also the target of the last original contribution on fiber designs in this special issue: Geometry of Chalcogenide Negative Curvature Fibers for $\mathrm{CO}_{2}$ Laser Transmission [16] by the Baylor University and the University of Maryland Baltimore County, in Baltimore (USA). In this paper, a large number of geometrical parameters are used in numerical simulations on HCs in chalcogenide glasses, in order to achieve the best possible attenuation performances at the $\mathrm{CO}_{2}$ laser wavelength of $10.6 \mu \mathrm{m}$. This numerical work is of high importance in the field since anti-resonant HCs could be a valid alternative to other types of specialty optical fibers for the mid-infrared spectral range.

For example, passing now to the original experimental contribution of this special issue, the paper Fabrication of Shatter-Proof Metal Hollow-Core Optical Fibers for Endoscopic Mid-Infrared Laser Applications [17] by the Sendai College, the Miyagi Gakuin Women's University, the Tohoku University, in Japan, and the Fudan University, in China, concerns the experimental demonstration of innovative HCs for the $10.6 \mu \mathrm{m}$ wavelength. Targeting medical applications of HCs, this paper addresses relevant implementation issues of this technology by looking, in particular, not only at the fiber attenuation and bending loss, but also at the characteristics of the material embedded inside the $\mathrm{HC}$ and at the ability of the same HC in guiding both mid-infrared and visible light for its practical operation.

Practicability in the device implementation is also the object of the second original experimental contribution to this issue. The paper Combining Hollow-core Photonic Crystal Fibers with Multimode, Solid Core Fiber Couplers through Arc Fusion Splicing for the Miniaturization of Nonlinear Spectroscopy Sensing Devices [18], by the Wroclaw University of Science and Technology, in Wroclaw (Poland), deals with the important problem of combining HCs and standard optical technology, in an effective and viable way. The optimization of the splicing parameters, by simply using a conventional arc fusion splicer, allows them to demonstrate adequate performances and the validity of their approach in a two-photon fluorescence spectroscopy experiment.

On the other hand, a method to process $\mathrm{HCs}$ via a $\mathrm{CO}_{2}$ laser is used in the third original experimental work of this issue. The paper A Method to Process Hollow-Core Anti-Resonant Fibers into Fiber Filters [19], by the Nanyang Technological University, discusses how to modify the internal geometrical characteristics of an anti-resonant $\mathrm{HC}$ in order to use it as a filter device. It shows how the implemented methodology could also be employed in the dispersion control, a very relevant factor in optical pulse propagation and manipulation.

The study of the characteristics of high-power pulses delivered through an $\mathrm{HC}$ is the thematic of the last original experimental contribution to this special issue. The paper Hollow-core Optical Fibers for Industrial Ultra Short Pulse Laser Beam Delivery Applications [20], by Photonic Tools GmbH, in Berlin, shows relevant details on the implementation of a high-power laser-beam delivery device, in both the picosecond and femtosecond pulse duration regime. The suitability of HCs for flexible and efficient optical-power delivery was proved by the results when cutting different materials.

Besides providing a good balance between reviews (3 contributions), theoretical analysis ( 3 contributions) and applications (4 contributions), this special issue of Fibers represents a reasonable mix of the research activities from different geographical areas, with contributions from the European 
Union (5 research institutions and 2 companies), Russia (1 institution), Brazil (3 institutions), United States of America (2 institutions), Japan (2 institutions), China (1 institution) and Singapore (1 institution). This shows the worldwide interest for a technology that is coming to better maturity and may largely affect industrial, economical and societal changes in the future years.

Funding: This research received no external funding.

Conflicts of Interest: The authors declare no conflict of interest.

\section{References}

1. Marcatili, E.; Schmeltzer, R. Hollow Metallic and Dielectric Waveguides for Long Distance Optical Transmission and Lasers. Bell Syst. Tech. J. 1964, 43, 1783-1809. [CrossRef]

2. Cregan, R.F.; Mangan, B.J.; Knight, J.C.; Birks, T.A.; St. J. Russell, P. Single-Mode Photonic Band Gap Guidance of Light in Air. Science 1999, 285, 1537-1539. [CrossRef] [PubMed]

3. Venkataraman, N.; Gallagher, M.T.; Smith, C.M.; Muller, D.; West, J.A.; Koch, K.W.; Fajardo, J.C. Low Loss $(13 \mathrm{~dB} / \mathrm{km})$ Air Core Photonic Band-Gap Fibre. In Proceedings of the ECOC 2002, Copenhagen, Denmark, 8-12 September 2002. PD1.1.

4. Roberts, P.J.; Couny, F.; Sabert, H.; Mangan, B.J.; Williams, D.P.; Farr, L.; Mason, M.W.; Tomlinson, A.; Birks, T.A.; Knight, J.C.; et al. Ultimate low loss of hollow-core photonic crystal fibres. Opt. Express 2005, 13, 236-244. [CrossRef] [PubMed]

5. Lombardini, A.; Mytskaniuk, V.; Sivankutty, S.; Ravn Andresen, E.; Chen, X.; Wenger, J.; Fabert, M.; Joly, N.; Louradour, F.; Kudlinski, A.; et al. High-resolution multimodal flexible coherent Raman endoscope. Light Sci. Appl. 2018, 7, 10. [CrossRef] [PubMed]

6. Cruz, A.; Serrão, V.A.; Barbosa, C.L.; Franco, M.A.R.; Cordeiro, C.M.B.; Argyros, A.; Xiaoli, T. 3D Printed Hollow Core Fiber with Negative Curvature for Terahertz Applications. J. Microw. Optoel. Electromagn. Appl. 2015, 14, 45-53.

7. Wang, X.; Ge, D.; Ding, W.; Wang, Y.Y.; Gao, S.; Zhang, X.; Sun, Y.; Li, J.; Chen, Z.; Wang, P. Hollow-core conjoined-tube fiber for penalty-free data transmission under offset launch conditions. Opt. Lett. 2019, 44, 2145-2148. [CrossRef]

8. Michieletto, M.; Lyngsø, J.K.; Jakobsen, C.; Lægsgaard, J.; Bang, O.; Alkeskjold, T.T. Hollow-core fibers for high power pulse delivery. Opt. Express 2016, 24, 7103-7119. [CrossRef] [PubMed]

9. Digonnet, M.J.F.; Chamoun, J.N. Recent developments in laser-driven and hollow-core fiber optic gyroscopes. Proc. SPIE 2016, 9852, 985204.

10. Giovanardi, F.; Cucinotta, A.; Rozzi, A.; Corradini, R.; Benabid, F.; Rosa, L.; Vincetti, L. Hollow Core Inhibited Coupling Fibers for Biological Optical Sensing. J. Light. Technol. 2019, 37, 2598-2604. [CrossRef]

11. Debord, B.; Amrani, F.; Vincetti, L.; Gérôme, F.; Benabid, F. Hollow-Core Fiber Technology: The Rising of "Gas Photonics". Fibers 2019, 7, 16. [CrossRef]

12. Bufetov, I.A.; Kosolapov, A.F.; Pryamikov, A.D.; Gladyshev, A.V.; Kolyadin, A.N.; Krylov, A.A.; Yatsenko, Y.P.; Biriukov, A.S. Revolver Hollow Core Optical Fibers. Fibers 2018, 6, 39. [CrossRef]

13. Cruz, A.L.S.; Cordeiro, C.M.B.; Franco, M.A.R. 3D Printed Hollow-Core Terahertz Fibers. Fibers 2018, 6, 43. [CrossRef]

14. Provino, L. Effect of Nested Elements on Avoided Crossing between the Higher-Order Core Modes and the Air-Capillary Modes in Hollow-Core Antiresonant Optical Fibers. Fibers 2018, 6, 42. [CrossRef]

15. Zeisberger, M.; Hartung, A.; Schmidt, M.A. Understanding Dispersion of Revolver-Type Anti-Resonant Hollow Core Fibers. Fibers 2018, 6, 68. [CrossRef]

16. Wei, C.; Menyuk, C.R.; Hu, J. Geometry of Chalcogenide Negative Curvature Fibers for $\mathrm{CO}_{2}$ Laser Transmission. Fibers 2018, 6, 74. [CrossRef]

17. Iwai, K.; Takaku, H.; Miyagi, M.; Shi, Y.W.; Matsuura, Y. Fabrication of Shatter-Proof Metal Hollow-Core Optical Fibers for Endoscopic Mid-Infrared Laser Applications. Fibers 2018, 6, 24. [CrossRef]

18. Stawska, H.I.; Popenda, M.A.; Bereś-Pawlik, E. Combining Hollow Core Photonic Crystal Fibers with Multimode, Solid Core Fiber Couplers through Arc Fusion Splicing for the Miniaturization of Nonlinear Spectroscopy Sensing Devices. Fibers 2018, 6, 77. [CrossRef] 
19. Huang, X.; Yong, K.T.; Yoo, S. A Method to Process Hollow-Core Anti-Resonant Fibers into Fiber Filters. Fibers 2018, 6, 89. [CrossRef]

20. Eilzer, S.; Wedel, B. Hollow Core Optical Fibers for Industrial Ultra Short Pulse Laser Beam Delivery Applications. Fibers 2018, 6, 80. [CrossRef]

(C) 2019 by the author. Licensee MDPI, Basel, Switzerland. This article is an open access article distributed under the terms and conditions of the Creative Commons Attribution (CC BY) license (http://creativecommons.org/licenses/by/4.0/). 